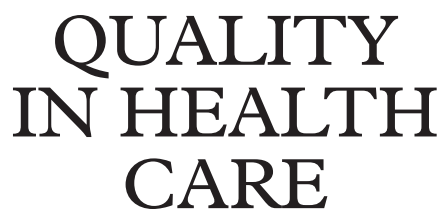

Editorials

\title{
Assessment of quality of life as an outcome: finding measurements that reflect individuals' priorities
}

The concept of quality of life is increasingly recognised and used as an important dimension of measurement of outcome in clinical trials and other forms of evaluative research. One criticism sometimes voiced of the questionnaires and interview schedules purporting to assess this aspect of patients' experiences is that by adopting a standardised content in terms of questionnaire items and scoring of responses, the vast majority of instruments fail to address the diversity of priorities and concerns of respondents and the varying weights and values which they attach to their concerns. Several groups have developed instruments intended to overcome what might appear to be fundamental limitations in standardised quality of life instruments.

The MACTAR (McMaster-Toronto Arthritis and Rheumatism Questionnaire) patient preference disability questionnaire for use in clinical trials in arthritis was developed by Tugwell et al over 10 years ago. ${ }^{1}$ At base line, respondents nominate up to five activities affected by arthritis. At follow up they rate the extent of improvement in each nominated activity. In one trial of a drug for arthritis, this "individualised" measure was more sensitive to changes experienced by patients than conventional standardised measures of outcome. ${ }^{2}$ The authors suggest that one reason for greater sensitivity is that questionnaire items not of concern to patients create "noise" in conventional standardised instruments. A similar instrument, known as the schedule for the evaluation of individual quality of life (SEIQoL), was developed for use across the full spectrum of health problems. ${ }^{3}$ The individual identifies up to five domains important to quality of life. Respondents also have to complete tasks in the form of judgments about case vignettes to derive the weights that respondents attach to nominated areas of quality of life. Because respondents face no constraints in areas of life they may nominate, the instrument assesses a much broader construct than the MACTAR disability questionnaire. Its authors consider SEIQoL to be maximally sensitive to individuals' personal quality of life and have shown sensitivity to change in the quality of life of patients undergoing total hip replacement surgery. ${ }^{4}$

In this issue of Quality in Health Care, Ruta et al report evidence of measurement properties of the patient generated index, an instrument developed with a similar philosophy to those cited above. ${ }^{5}$ Essentially, respondents are asked to choose areas of life affected by a particular health problem and then assign numerical values to express severity of impact and personal importance of areas chosen. Unlike the SEIQoL, the version of the patient generated index evaluated in this report is designed for self completion in a postal questionnaire. The attempt to assess quality of life in terms relevant to individuals' personal concerns by means of the practical method of a self complete questionnaire is an important contribution because it acknowledges that outcome assessment almost always has to be integrated into time and resource constraints of routine clinical care.

Such instruments raise various important issues about how patients should best participate in the evaluation of outcomes of their health care. Firstly, the field needs to become clearer and more explicit about concepts and constructs that are to be measured. The patient generated index is presented as a measure of quality of life, but the format of the instrument, by focusing on the impact on the individual of a health problem, focuses on the narrower domain of health related quality of life. The distinction is not purely semantic because the broader construct of quality of life encompasses issues such as life satisfaction not readily assessed by the patient generated index. ${ }^{6}$

The authors of the patient generated index have carefully provided evidence to assess the instrument's psychometric properties of reproducibility, construct validity, and responsiveness. A full evaluation of an instrument's usefulness, however, requires evidence about its acceptability and the feasibility of its use in specific settings. ${ }^{7}$ Some patients, particularly the less advantaged, have difficulties understanding the patient generated index. This may explain the disappointing rate at which respondents complete and return it. If it proves to be the case that instruments such as the patient generated index and SEIQoL have to be personally given by interview to achieve satisfactory response rates, this becomes an important consideration in assessing feasibility in large scale multicentre evaluations. The greater claimed validity and precision of more indepth individualised instruments is not without cost, and more evidence is needed of the tradeoffs between validity on the 
one hand and practical feasibility and acceptability on the other hand.

Another criterion which needs to be more widely applied to patient based outcome measures is interpretability. ${ }^{7}$ Here we are concerned with the question of how meaningful are results produced by an instrument to diverse audiences such as clinicians, commissioners, and consumer groups. Difficulties exist that need to be addressed to make any patient assessed outcome measure meaningful ("what does a 10 point improvement in this mobility scale really mean?"), especially while lack of routine use inhibits the development of familiarity. It is not clear whether individualised measures face additional problems of interpretability in that, for groups of patients, the issue is a 10 point improvement in a dimensionless category.

In their interesting study, Ruta et al raise a fundamental issue with regard to individualised and standardised instruments. They consider the patient generated index currently unsuitable for use in individual patients because of measurement error, while paradoxically noting its potential use as a management tool in patient care. More evidence is needed of the value of conventional as well as individualised patient based outcome measures in individual patient care. By comparison, a clearer potential role has been demonstrated for such instruments in randomised controlled trials where often the main matters of concern to patients are measured and the issue of attribution of outcomes to intervention is addressed by randomisation.
The study by Ruta et al shows the way forward for this field. Greater effort now needs to be invested in the comparative evaluation, against a full range of criteria, of the performance of different types of instruments either in dedicated methodological studies or as "bolt on" components of trials and other evaluative studies of the outcomes of healthcare interventions.

\section{RAY FITZPATRICK}

Professor of Public Health and Primary Care, Institute of Health Sciences,

University of Oxford,

Oxford, UK

1 Tugwell P, Bombardier C, Buchanan W, et al. The MACTAR patient preference disability questionnaire - an individualized functional priority approach for assessing improvement in physical disability in clinical trials in proach for assessing improvement in physical disab
rheumatoid arthritis. $\mathcal{F}$ Rheumatol $1987 ; \mathbf{1 4}: 446-51$.

2 Tugwell P, Bombardier C, Buchanan W, et al. Methotrexate in rheumatoid arthritis: impact on quality of life assessed by traditional standard item and individualized patient preference health status questionnaires. Arch Intern Med 1990;150:59-62.

3 Browne J, O'Boyle C, McGee H, et al. Individual quality of life in the healthy elderly. Qual of Life Res 1994;3:235-44.

4 O'Boyle C, McGee H, Hickey A, et al. Individual quality of life in patients undergoing hip replacement. Lancet 1992;339:1088-91.

5 Ruta D, Garratt A, Russell I. Patient-centred assessment of quality of life for patients with four common conditions. Quality in Health Care

6 Kreitler S, Chaitchik S, Rapoport Y, et al. Life satisfaction and health in cancer patients, orthopedic patients and healthy individuals. Soc Sci Med 1993; 36:547-56.

7 Fitzpatrick R, Davey C, Buxton M, et al. Evaluating patient-based outcome measures for use in clinical trials. Health Technol Assessment 1998;2:1-74. 


\section{Continuous quality improvement: a solution for primary care and care across the interface?}

It wasn't that there was one particular problem, or that there was a disastrous breakdown in care, but it seemed to Mrs $\mathrm{H}$ that during the final stages of her husband's illness, the healthcare team supporting them in their own home gave the impression of being in a muddle. Messages did not get through efficiently, prescriptions were never ready for collection at the times arranged, and the staff who visited her husband, although sympathetic, did not seem to have a coherent plan, so that treatment was changed at frequent and confusing intervals without evident benefit. Consequently, Mrs $\mathrm{H}$ became used to reminding the team about its undertakings, and her expectations of them were adjusted downwards. It felt to her as though the team did not fully care about her predicament and her husband's suffering.

No matter how hard we try as individuals to provide the highest quality care, if the system in which we work conspires against us, the end result is often depressing. Just as Mrs $\mathrm{H}$ in the case study above, patients become disillusioned about healthcare services and a spiral of recrimination and deteriorating professional and patient relationship takes over.

To achieve quality care we need not only quality professionals but also quality systems within which they work. Continuous quality improvement programmes may be one approach to improving the quality of both systems and individuals. A growing number of successful examples exist of continuous quality improvement in hospital practice from Europe and North America. There are few similar examples from primary health care, however, ${ }^{1}$ and even fewer examples that address the problems of intersectoral and interdisciplinary cooperation, even though patients may feel in limbo during their passage through the healthcare system. ${ }^{2}$ The need for quality development at the interface between primary and secondary care is especially important as patients with chronic diseases often cross to and fro between hospital and primary care and they are often supported by teams from both sectors.

In many countries, primary health care is provided by multiprofessional teams who collaborate and have some responsibility for managing themselves. Such teams could be fertile soil for the introduction of continuous quality improvement within the practice and at the interface with other parts of the healthcare system. There are early signs that such an approach could be widely implemented. In Spain and Portugal, programmes for continuous quality improvement in primary care have been set up and early experiences have been promising. ${ }^{3}$ Developments in these countries have had widespread support and commitment from the top of the healthcare organisation. In other countries, demonstration or experimental projects have been undertaken. For example, in the United States a trial of quality improvement in implementing guidelines was done, but the results were disappointing. ${ }^{4}$ In Europe, several observational studies have been done. Lawrence et al showed how quality improvement techniques could be introduced to primary care teams through training and workshops followed up by continued support. ${ }^{5}$ Experiences with facilitators in the Netherlands and United Kingdom also showed that they may be a practical way to implement continuous quality improvement for handling specific diseases and problems. ${ }^{6}$ Different shared care programmes for chronic diseases also suggest that there may be a potential for continuous quality development of interface problems. ${ }^{78}$

Supporting the introduction of continuous quality improvement could be expensive in time and money, and evidence is needed therefore about its long term cost effectiveness in relation to other activities. However, there are several obstacles to research into continuous quality improvement in primary care. The first is that a clearly defined and replicable model of continuous quality improvement is required. In the studies that have been undertaken thus far, different models have been used and it is difficult to judge whether any differences between outcomes are due to the model or other factors. Secondly, if continuous quality improvement is to be widely applied, it must be tested by a wide variety of practices and primary care teams. Its effectiveness should be demonstrated for many types of problems, including those found at the interface between primary and secondary care. Thirdly, we need to know whether effectiveness varies in different types of teams and practice settings, and when different quality problems in primary care are addressed whether they relate to the core content of medical care, communication, continuity of care, or coordination of care across the interface. Fourthly, we need to know whether the observed benefits persist over a long period of time, or whether continued support is required by the participating teams.

Geboers et al report in this issue of the journal a study that begins to address some of these questions and takes the issue of quality improvement for primary healthcare teams a step further. ${ }^{9} 10$ They have developed a clearly defined model of continuous quality improvement suitable for the types of practices common in the Netherlands, including single handed practices. The core elements of their model are appropriate management of the team, factual data for guiding and monitoring quality improvement, a systematic approach for quality improvement methods, and collaboration among team members. They showed the feasibility of their approach and that quality improvements could be achieved, but the application of the model tended to disintegrate once facilitator support had been withdrawn.

There are clear messages for policy makers. Continuous quality improvement in primary health care can be achieved and it can be associated with improved performance. Its introduction requires commitment and motivation from policy makers and managers of health services. It is likely to require long term support rather than being a short programme that will rapidly become self sustaining.

The Dutch study also draws attention to major remaining questions for research, including how to develop the total healthcare system based on evidence from organisational research. One urgent priority is large long term randomised controlled trials with interventions based on solid theory, for instance the model of continuous quality improvement. Such trials should address quality improvement within the practice and at the interface with other parts of the healthcare system. Measures of effectiveness should include a mix of medical outcome measures, patients' evaluations, and cost/effect estimations. Only studies of this type can help us to decide whether the 
continuous quality improvement approach is the way forward for improving both primary and secondary care and the interface between them. Healthcare systems in Europe must find the commitment to tackle these important questions.

RICHARD BAKER

Director, Eli Lilly National Clinical Audit Centre, Leicester, UK

FREDE OLESEN

Research director, Research Unit for General Practice, Aarhus University, Denmark

1 Soberg LI, Brekke ML, Kottke TE, et al. Continuous quality improvement in primary care: what's happening? Medical Care 1998;5:625-53.

2 Preston C, Cheater F, Baker R, et al. Left in limbo: patients' views on care across the primary/secondary interface. Quality in Health Care 1999;
3 Grol R, Baker R, Roberts R, et al. Systems for quality improvement in general practice. A survey in 26 countries. Eur $\mathcal{F}$ Gen Pract 1997;3:65-8.

4 Goldberg HI, Wagner EH, Fihn SD, et al. A randomized controlled trial of CQI teams and academic detailing: can they alter compliance with guidelines? I Quality Improvement 1998;24:130-42.

5 Lawrence M, Packwood T. Adapting total quality management for general practice: evaluation of a programme. Quality in Health Care 1996;5:151-8.

6 Hearnshaw $\mathrm{H}$, Reddish S, Carlyle D, et al. Intorducing a quality improvement programme to primary healthcare teams. Quality in Health Care 1998; 7:200-8.

7 Pritchard P, Hughes J. Shared care: the future imperative? London: The Royal Society of Medicine Press Ltd, 1996.

8 Hulscher MEJL. Implementing prevention in general practice: a study on cardiovascular disease. Centre for Quality of Care Research, Universities of Nijmegen and Maastricht, 1995.

9 Geboers H, Grol, van den Bosch W, et al. A model for continuous quality improvement in small scale practices. Quality in Health Care 1999;8:43-8.

10 Geboers H, van der Horst M, Mokkink H, et al. Setting up improvement projects in small scale primary care practices: feasibility of a model for continuous quality improvement. Ouality in Health Care 1999;8:36-42.

\section{Journal scan: quality improvement at a glance}

Quality improvement is not a specialist subject. Useful papers on the techniques and successes of quality improvement are published in many healthcare journals and have interest beyond the main professional or specialist interest of the journal. As more journals are published electronically, such papers will be easily accessed by a wide readership. But, for now, we mostly read paper journals, and tend to confine our reading to specialist and professional journals within our tribes. And so, in this issue of Quality in Health Care we include for the first time a selection of abstracts of papers relevant to quality improvement, chosen by two abstracters from recent issues of three healthcare journals (BMF, Lancet, and $\mathcal{F} A M A)$ and from three nursing journals (International fournal of Nursing Studies, Fournal of Advanced Nursing, and Fournal of Nursing Care Quality). The aim is to give readers easy access to a wider range of writing and research on quality improvement. Fournal Scan will be a regular feature of this journal. By scanning only six journals we have started modestly. We selected well known journals. As we gain experience we hope to scan more journals including journals from the rest of Europe. We are interested in readers' views on this new section and in suggestions for journals we should scan.

FIONA MOSS 


\section{Patient education in the year 2000: tailored decision support, empowerment, and mutual aid}

Giving patients easy access to information about their health and their health care is one characteristic of good quality care. The emergence of a better educated and more consumer aware population and increasing interest in consumer based strategies to promote appropriate utilisation of health resources has raised awareness of the importance of patients being involved in their own care. If patients are to make decisions about their own health care, however, information alone may not be enough; patients also need to be supported through the process of making choices, particularly if they are facing life threatening chronic illness.

The critieria that describe good counselling about health issues have recently been considered by the US National Committee on Quality Assurance. These include the importance of knowing about all options, benefits, and harms; having the opportunity to participate in decision making; and to convey preferences to their practitioners. This initiative recognised not only the importance of defining and monitoring the quality of counselling but also that of identifiying practical tools that practitioners can use to enhance counselling about complex decisions.

Through development of information technology, information that once "belonged" to professionals is now widely accessible. And from work of the evidence-based practice movement, information about clinical interventions previously "hidden" away in journals on library shelves has been re-analysed in systematic reviews and meta-analyses and repackaged into clinically relevant formats. Thus the tools to allow patients to be real partners in deciding the course of their health care are available and their use may soon become the norm. However, although considering informing patients and involving them more fully in their own care is important, we must not forget that the tools and processes used to support patients in this should themselves be subjected to critical assessment. Development and evaluation of patient informatics tools is an area of research that will soon influence routine clinical practice. A review commissioned by the US Agency for Health Care Policy summarised the conceptual and methodological challenges in this area and, drawing on studies from Canada, the UK, and the US, identified research priorities. ${ }^{1}$ These include assessment of the impact of informatics tools on a wide range of patient outcome measures; identification of factors that influence patient use of these tools; assessment of the effects of these tools on patient-clinical communication; and evaluation of cost effectiveness.

Research into some of these issues is already underway and the potential benefits of computer access to both information and to support are becoming clearer.

In this issue of Quality in Health Care, Gustafson and a team from University of Wisconsin-Madison, pioneers in this research, summarise some of the findings from their research and development into the Comprehensive Health Enhance Support System (CHESS). This is a multiservice computer system that provides not only information but also a means of support to people facing complex decisions and uncertainty. The results are compelling. There are clear benefits to patients, and there must be a role for interventions such as CHESS. But using these systems is not straightforward and there are many questions still to be answered. Trials in progress will establish the generalisability of these findings from their promising early work. As the experience in the use of these systems increases we will need to establish which support strategies work best for different groups of patients. Moreover, we need to discover which components in the "black box" of our support are most efficacious and efficient. Some components are expensive and complex, and we need to be sure of their added value. For example, CHESS uses a multi-attribute utility approach to assist women in deciding about breast cancer surgery options. Other decision supports have been developed using technologies ranging from simple pamphlets to interactive video disks. ${ }^{2-4}$ As two studies suggest that providing brief information on options and outcomes in pamphlets may work just as well as the more complicated decision support tools, ${ }^{45}$ we may need to reconsider the strategies we should be using to deliver decision support. Similar questions can be posed regarding other labour intensive support strategies provided by informatics tools, such as "ask an expert".

Many informatics tools are developed based on academic expertise and interest. However, development of clinical applications for use in routine practice should be based on needs assessment. For which types of conditions are support tools most needed? How can we ensure that these tools are developed, evaluated, disseminated, and updated? What standards can be developed to ensure that consumers have access to tools that are evidence-based, balanced, up to date, efficacious, and efficient? What are the most effective dissemination strategies? The careful evolution of the work of Gustafson et al is a model for the development of patient informatics tools. It also highlights the importance of providing timely, tailored, interactive, and evidence-based information and the potential benefits of decision support and mutual aid systems. As we begin to consider these issues, it becomes obvious that this is an area of research that has enormous potential but one that is only just beginning.

ANNETTE M O'CONNOR,

Professor and Ontario Ministry of Health Career Scientist, University of Ottawa School of Nursing and Faculty of Medicine, Ottawa, Ontario, Canada

1 Hersey J, Matheson J, Lohr K.Consumer health informatics and patient decision-making. (AHCPR Pub No 98-N001). Rockville, MD: Agency for decision-making. (AHCPR Pub No 98-NOD

2 Kasper JF, Mulley AG Jr, Wennberg JE. Developing shared decision making programs to improve the quality of health care. Quality Review Bulletin 1992;18:183-90.

3 Sawka CA, Goel V, Mahut CA, et al. Development of a patient decision aid for choice of surgical treatment for breast cancer. Health Expectations 1998; $1: 23-36$

4 Goel V, Sawka C, Thiel E, et al. A randomised trial of a decision aid for breast cancer surgery (abstract). Medical Decision Making 1998;18:482.

5 Rothert ML, Holmes-Rovner M, Rovner D, et al. An educational intervention as decision support for menopausal women. Research in Nursing and Health 1997;20:377-87. 